\section{Anti-inflammatory angle in obesity}

\section{By Lev Osherovich, Senior Writer}

An international team has discovered a Wnt signaling pathway in white adipose tissue that could be manipulated to short-circuit obesity-associated inflammation and thus potentially prevent metabolic syndrome and type 2 diabetes. ${ }^{1}$ The findings may open a new therapeutic area for the few drug developers in the Wnt space if it can be targeted without triggering side effects in other tissues.

Although the Wnt pathway has been studied most extensively in embryonic development and cancer, a role for the pathway in adipose tissue has been hinted at in previous studies. ${ }^{2}$ The new study now identifies a mechanism by which an adipocyte-derived protein called secreted frizzled-related protein 5 (SFRP5) blocks the activity of a proinflammatory Wnt pathway activator known as wingless-type MMTV integration site family member 5A (WNT5A).

WNT5A triggers macrophage activation, an early step in inflammation.

"We've identified a new therapeutic target system" for obesity-related inflammation and associated diseases, said Kenneth Walsh, professor of medicine and director of the Whitaker Cardiovascular Institute at the Boston University School of Medicine. Walsh led the team behind
"If modulating this pathway could induce an oncogenic signal, this will be a problem."

-Alan Solinger, Xoma Ltd. insulin response than overfed wild-type mice or $S f r p 5$ knockouts on a normal diet.

Having established a link between SFRP5 dysfunction and metabolic syndrome, the next step was uncovering a mechanism for the adipocyte-derived protein's protective functions.

The team found that Sfrp5 knockouts had abnormally high activity in a branch of the Wnt signaling pathway previously known to be overactive in diabetes. ${ }^{4}$

Walsh's group went on to show that WNT5A and SFRP5 have opposite effects on inflammatory signaling in cultured macrophages. WNT5A treatment increased the production of proinflammatory cytokines compared with no treatment; however, exposing the cells to SFRP5 blocked the effect of WNT5A.

Finally, obese mice overexpressing Sfrp5 had less adipose tissue inflammation, insulin resistance and fat accumulation in the liver than vector-treated controls.

Although the size of adipose cells was somewhat reduced in Sfrp5overexpressing mice, "the effects of this pathway on weight gain or loss are minor compared with its effect on inflammation," said Walsh. "When we overexpress SFRP5, the fat becomes less inflamed."

\section{Against the Wnt}

Altogether, the findings in the Science paper suggest that under normal circumstances "SFRP5 is produced by adipocytes and pacifies the macrophages to keep them working normally," said Walsh. In obesity, adipocytes fail to produce SFRP5, and as a result, the proinflammatory signal triggered by WNT5A predominates, he told SciBX.

Alan Solinger, VP of clinical immunology at Xoma Ltd., said Walsh's study points to an active role for adipocytes in influencing the inflammatory environment. He thinks the findings belie the conventional perception the study, which was published in Science.

The challenge now is to sort out how the inflammatory cascade that is stimulated by WNT5A and blocked by SFRP 5 fits in with other inflammatory mechanisms that go awry in obesity.

\section{Balancing act}

Walsh's team came across SFRP5 in a prior study of adipocyte-specific gene expression. ${ }^{3}$ In the new study, the researchers characterized SFRP5 expression in healthy and obese rodents and humans.

Overfed or genetically diabetic mice and rats had lower Sfrp5 levels than normally fed or healthy controls. Likewise, adipose tissue biopsies from obese individuals showed a correlation between low levels of SFRP5 and high levels of inflammatory cytokines and activated macrophages, which precede the low-grade inflammation that often accompanies metabolic syndrome.

These findings suggested that SFRP5 could keep the inflammatory component of metabolic syndrome in check. Indeed, the researchers found that overfed Sfrp 5 knockout mice had more macrophage activity and inflammatory cytokines as well as lower glucose tolerance and that adipocytes are inert storage depots for lipids.

SFRP5 and WNT5A appear to be elements of the "yin and yang" of cross-talk between adipocytes and nearby innate immune cells that keeps inflammation in check. In type 2 diabetes, "it seems that SFRP5 at some point is overcome, leading to inflammation," said Solinger.

Xoma's XOMA-052, a mAb that blocks the inflammatory cytokine IL-1 $\beta$, is in Phase II testing to treat type 2 diabetes.

Solinger said it remains unclear where SFRP5 and WNT5A fit into the cascade of immunological events in obesity-associated inflammation. Testing the effect of IL-1 $\beta$ blockers like XOMA-052 on WNT5A activity and the effect of SFRP5A treatment on IL- $1 \beta$ response could help sort this out, he noted.

Hans-Michael Dosch, professor of immunology at the University of Toronto and senior scientist at The Hospital for Sick Children, wanted to see how SFRP5 and WNT5A affect other immune cells involved in obesity-associated inflammation.

Last year, Dosch's team reported that the adipose tissue of obese 


\section{ANALYSIS}

\section{TARGETS \& MECHANISMS}

mice had abnormally high levels of T helper type 1 (Th1) cells and low levels of $\mathrm{T}_{\text {reg }}$ cells. ${ }^{5}$

"It would be interesting to see if their Sfrp5 knockout develops Th1 cell infiltration, a critical factor toward progression" from obesity to metabolic syndrome and diabetes, said Dosch.

\section{CrossWnts}

Walsh's study suggests that either increasing SFRP5 activity or blocking WNT5A could be a therapeutic strategy to arrest progression to metabolic syndrome.

One idea is to treat obese individuals with SFRP5, "but you might also target WNT5A with an antibody or target its receptor with a small molecule antagonist," he said. "It's not clear to me which would be the best way at this point."

Solinger said his biggest reservation about targeting SFRP5 and WNT5A is the Wnt pathway's involvement in tumor growth in other tissues. Whereas SFRP5 appears to be predominantly active in adipose tissue, WNT5A controls the development of a range of other tissue types and has been implicated in melanoma. ${ }^{6}$

Because many receptors and signaling components are shared by Wnt pathways and are at work in various cell types, perturbing either SFRP5 or WNT5A could have undesirable knock-on effects, Solinger told SciBX.
"The cancer connection is a red flag to many companies," he said. "If modulating this pathway could induce an oncogenic signal, this will be a problem."

Walsh's next step is to test whether long-term SFRP5 treatment in rodents can ultimately reverse metabolic syndrome. He has personally patented the findings and said the IP is available for licensing.

Osherovich, L. SciBX 3(26); doi:10.1038/scibx.2010.786

Published online July 1, 2010

\section{REFERENCES}

1. Ouchi, N. et al. Science; published online June 17, 2010; doi:10.1126/science. 1188280

Contact: Kenneth Walsh, Boston University School of Medicine, Boston, Mass.

e-mail: kxwalsh@bu.edu

Contact: Noriyuki Ouchi, same affiliation as above e-mail: nouchi@bu.edu

2. Christodoulides, C. et al. Trends Endoc. Metab. 20, 16-24 (2008)

3. Izumiya, Y. et al. Cell Metab. 7, 159-172 (2008)

4. Hirosumi, J. et al. Nature 420, 333-336 (2002)

5. Winer, S. et al. Nat. Med. 15, 921-929 (2009)

6. Weeraratna, A.T. et al. Cancer Cell 1, 279-288 (2002)

\section{COMPANIES AND INSTITUTIONS MENTIONED}

Boston University School of Medicine, Boston, Mass. The Hospital for Sick Children, Toronto, Ontario, Canada University of Toronto, Toronto, Ontario, Canada Xoma Ltd. (NASDAQ:XOMA), Berkeley, Calif. 